\title{
Activity-dependent polyadenylation in neurons
}

\author{
LING DU and JOEL D. RICHTER \\ Program in Molecular Medicine, University of Massachusetts Medical School, Worcester, Massachusetts 01605, USA
}

\begin{abstract}
Activity-dependent changes in protein synthesis modify synaptic efficacy. One mechanism that regulates mRNA translation in the synapto-dendritic compartment is cytoplasmic polyadenylation, a process controlled by CPEB, the cytoplasmic polyadenylation element (CPE)-specific RNA binding protein. In neurons, very few mRNAs are known CPEB substrates, and none appear to be responsible for the effects on plasticity that are found in the CPEB knockout mouse. These results suggest that the translation of other mRNAs is regulated by CPEB. To identify them, we have developed a functional assay based on the polyadenylation of brain-derived mRNAs injected into Xenopus oocytes, a surrogate system that carries out this $3^{\prime}$ end processing event in an efficient manner. The polyadenylated RNAs were isolated by binding to and thermal elution from poly(U) agarose and identified by microarray analysis. Selected sequences that were positive for polyadenylation were cloned and retested for polyadenylation by injection into oocytes. These sequences were then examined for activity-dependent polyadenylation in cultured hippocampal neurons. Finally, the levels of two proteins encoded by polyadenylated mRNAs were examined in glutamate-stimulated synaptoneurosomes. These studies show that many mRNAs undergo activity-dependent polyadenylation in neurons and that this process coincides with increased translation in the synapto-dendritic compartment.
\end{abstract}

Keywords: CPEB; polyadenylation; translation; synaptic plasticity

\section{INTRODUCTION}

Experience-dependent changes at neuronal synapses probably underlie long-term memory storage (Miller and Mayford 1999; Hering and Sheng 2001). Such changes may require the establishment of a tag at a synapse once it is stimulated; synaptic efficacy is based on the recognition of this tag (Frey and Morris 1997; Martin et al. 1997). The nature of the tag is unknown, but for at least two forms of plasticity, the long-lasting phase of long term potentiation (L-LTP) and long term depression (LTD), protein synthesis in dendrites (Kang and Schuman 1996; Huber et al. 2000), possibly at synapses (Scheetz et al. 2000; Aakalu et al. 2001), is involved. Cytoplasmic polyadenylation is one mechanism that governs mRNA translation in neurons $(\mathrm{Wu}$ et al. 1998); it requires two $3^{\prime}$ UTR elements: the CPE and AAUAAA (Mendez and Richter 2001). CPEB phosphorylation initiates polyadenylation (Mendez et al. 2000), which stimulates translation by causing Maskin, a CPEB and eIF4E-interacting factor, to dissociate from eIF4E (the mRNA cap binding protein); this event allows eIF4G to

Reprint requests to: Joel D. Richter, University of Massachusetts Medical School, 373 Plantation St., Worcester, MA 01605, USA; e-mail: joel.richter@umassmed.edu; fax (508) 856-4289.

Article published online ahead of print. Article and publication date are at http://www.rnajournal.org/cgi/doi/10.1261/rna.2870505. bind eIF4E and initiate translation (Stebbins-Boaz et al. 1999; Cao and Richter 2002).

CPEB is detected at postsynaptic sites of neurons (Wu et al. 1998), where its phosphorylation upon NMDA receptor activation (Huang et al. 2002; Atkins et al. 2004) stimulates the polyadenylation and translation of CPE-containing $\alpha$ CaMKII mRNA (Wu et al. 1998). To assess whether CPEB is involved in synaptic plasticity, Alarcon et al. (2004) measured several electrophysiological parameters of Schaffer collateral CA1 neurons of the hippocampus from CPEB knockout mice and found that single $100-\mathrm{Hz}$ stimulation and single theta burst stimulation of long term potentiation (LTP) was impaired. Aplysia neurons also contain CPEB; when it was depleted from these cells by an antisense oligonucleotide, the maintenance of long-term facilitation (LTF) was abrogated (Si et al. 2003). Thus, in vertebrates and invertebrates, $\mathrm{CPEB}$ modulates some forms of synaptic plasticity.

In vertebrates, only $\alpha$ CaMKII (Wu et al. 1998) and tissue type plasminogen activator (tPA) (Shin et al. 2004) mRNAs are known to undergo activity-dependent polyadenylation. If CPEB exerted its influence on plasticity solely through these mRNAs, then the changes in synaptic strength observed in the CPEB and the $\alpha$ CaMKII and tPA knockout mice would be expected to be similar; however, such is not the case (Silva et al. 1992; Frey et al. 1996; Alarcon et al. 2004). These results suggest that CPEB may influence 
synaptic plasticity through other mRNAs. To begin to identify them, we developed an assay based on the polyadenylation of synthetic mRNAs derived from rat brain injected into Xenopus oocytes. The mRNAs, which were identified by microarray analysis following thermal elution from poly $(\mathrm{U})$ agarose, were shown to undergo activity-dependent polyadenylation in cultured hippocampal neurons; in at least two cases, their local translation was stimulated as well.

\section{RESULTS}

Because the same cis and trans elements appear to promote cytoplasmic polyadenylation in both Xenopus oocytes and mammalian neurons (Richter 2001), we used oocytes as an efficacious surrogate system to identify brain mRNAs that undergo this process in an activity-dependent manner. However, the PCR-based PAT assay that is often used to measure polyadenylation (Salles et al. 1994) proved to be inadequate for our purposes, and thus we employed an alternative method. Here, RNA is suspended in a solution containing 25\% formamide (which lowers the melting temperature between RNA duplexes) and applied to a poly(U) agarose beads. The beads were washed in buffer containing $25 \%$ formamide at $50^{\circ} \mathrm{C}$, which removes nonspecific RNAs and those with short poly(A) tails, usually less than $\sim 50 \mathrm{nt}$. Formamide-containing buffer at $60^{\circ} \mathrm{C}$ is then passed through the column, which elutes RNAs with poly $(\mathrm{A})$ tails greater than $\sim 55$ bases (Simon et al. 1996, based on Palatnik et al. 1981).
To test the efficacy of this method, the $3^{\prime}$ UTR of $\alpha$ CaMKII mRNA, which contains two CPEs (Wu et al. 1998), was injected into oocytes that were then treated with progesterone. The extracted RNA was mixed with poly $(\mathrm{U})$ agarose, washed at $50^{\circ} \mathrm{C}$, and eluted at $60^{\circ} \mathrm{C}$. Figure $1 \mathrm{~A}$ demonstrates that while the injected $\alpha$ CaMKII RNA was stable in all oocytes (total RNA), it bound poly(U) agarose only from progesteronetreated oocytes, indicating that it had undergone polyadenylation. We also analyzed $\alpha$ CaMKII RNA where the CPEs were mutated, which abrogates polyadenylation ( $\mathrm{Wu}$ et al. 1998). As before, all injected RNAs were equally stable (total RNA). When the chromatography procedure was performed with RNA from oocytes of three different frogs, the wild-type RNA was always detected in the $60^{\circ} \mathrm{C}$ elution. In oocytes from two of three frogs, the mutant RNA was not detected; a faint signal, however, was detected in oocytes from the third frog (Fig. 1A, bottom). These results demonstrate that chromatography on poly $(U)$ agarose followed by thermal elution can be used to identify mRNAs that gain a poly $(A)$ tail in a CPEdependent manner.

Figure $1 \mathrm{~B}$ outlines our approach to use this method to identify brain RNAs that undergo polyadenylation. Plasmid DNA was prepared from 23,300 bacterial colonies containing a rat cDNA library (oligo $(\mathrm{dT})$ primed and directionally cloned) that were grown on 25 plates. The DNA derived from each plate was linearized, transcribed, and the poly(A) tails removed by treatment with oligo(dT)/RNase $\mathrm{H}$. The RNAs were injected into oocytes that were then incubated
A

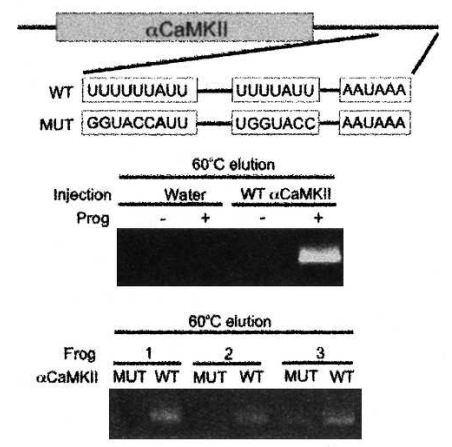

B

1.) Rat brain CDNA library in 25 pools containing $\sim 1000$ colonies

2.) Linearize plasmid DNA and Not $l$ and synthesize RNA

3.) Remove poly(A) tails with oligo(dT) and RNase $H$

4.) Inject RNAs into Xenopus oocytes

5.) Add progesterone to induce oocyte maturation

6.) Extract RNA, collect poly(A)+RNA on poly $(U)$ agarose beads; elute RNAs with long poly $(A)$ tails at $60^{\circ} \mathrm{C}$.

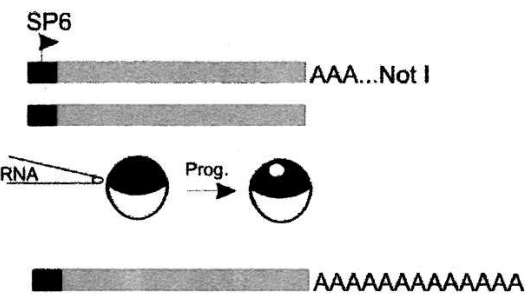

7.) Prepare biotin-labeled CRNA and probe rat brain microarray

8.) Determine the presence of CPE and AAUAAA

9.) Make individual transcripts in vitro and test for polyadenylation in injected oocytes

10.) Test for activity-dependent polyadenylation in neurons

FIGURE 1. Regimen for identifying brain mRNAs that undergo activity-dependent polyadenylation. (A) Schematic of $\alpha$ CaMKII mRNA denoting U-rich CPEs and polyadenylation hexanucleotide. A mutant $3^{\prime}$ UTR contains nucleotide substitutions in the CPEs (top). A portion of wild-type (WT) $\alpha$ CaMKII $3^{\prime}$ UTR was synthesized in vitro and injected into oocytes, some of which were treated with progesterone. The RNA was extracted and applied to poly $(\mathrm{U})$ agarose. The beads were washed at $50^{\circ} \mathrm{C}$ and the RNA eluted at $60^{\circ} \mathrm{C} ; \alpha$ CaMKII RNA was detected by RT-PCR (middle). Wild-type (WT) and mutant (MUT) $\alpha$ CaMKII $3^{\prime}$ UTRs were injected into oocytes from three frogs; the RNA was analyzed as above. (B) A rat brain cDNA library (oligo(dT)-primed, directionally cloned downstream of the SP6 promoter and polylinker) is spread onto 25 plates so that each contains $\sim 1000$ colonies. The colonies are collected, plasmid DNA is isolated, linearized with NotI, and transcribed. The poly(A) tails are removed by oligo(dT)/RNase H and the RNA is injected into Xenopus oocytes that are induced to mature with progesterone. RNA extracted from the oocytes is applied to poly $(\mathrm{U})$ agarose; the beads are washed at $50^{\circ} \mathrm{C}$ and the RNA eluted at $60^{\circ} \mathrm{C}$. The newly polyadenylated brain RNA from each plate is combined and used to make biotin-labeled cRNA for probing an Affymetrix neurobiology microarray (chip RN U34 containing $~ 1200$ sequences). Positive sequences are examined for the presence of a CPE and AAUAAA and some are then synthesized individually in vitro and retested for polyadenylation in injected oocytes. Some sequences are tested for activity-dependent polyadenylation in cultured hippocampal neurons. 
with progesterone, which stimulates meiotic maturation and cytoplasmic polyadenylation. The RNA was extracted and chromatographed on poly $(\mathrm{U})$ agarose as described above. The eluted RNA, which would contain both injected and endogenous RNAs that have long poly(A) tails, were reverse transcribed with oligo(dT) and PCR amplified with a primer complementary to a polylinker sequence present on the $5^{\prime}$ end of all injected mRNAs (it is this last step that distinguishes injected from endogenous sequences). The amplified products derived from the 25 plates were then combined and analyzed on a microarray. Of the 23,300 colonies obtained from the 25 plates, 447 were detected on the RN U34 chip (Affymetrix); 66 were determined to have undergone polyadenylation in injected oocytes $(\sim 15 \%)$ (see below). Analysis of the $3^{\prime}$ UTRs of these 66 RNAs showed that $40(61 \%)$ contained CPEs; $11(17 \%)$ did not have complete $3^{\prime}$ UTRs in the databases, so the identification of CPEs was not possible. Only 14 (21\%) contained no clear CPE (Tables 1 (summary) and 2).

To confirm that these RNAs indeed underwent polyadenylation in oocytes, the $3^{\prime}$ UTRs from several were cloned into vectors for in vitro transcription in the presence of ${ }^{32} \mathrm{P}$ UTP. Radiolabeled $3^{\prime}$ UTRs for ABP (AMPA binding protein), RCM3 (calmodulin), and ELK2 (ether a-go-go like 2, a potassium channel) were injected into oocytes, some of which were incubated with progesterone. The RNA was extracted and analyzed by a denaturing polyacrylamide gel. Figure 2A shows that these RNAs underwent progesterone-stimulated polyadenylation, as evidenced by retarded electrophoretic mobility; in two cases (ABP and RCM3), treatment with oligo(dT) and RNase $\mathrm{H}$ reversed the mobility change, thus confirming that these RNAs gained a poly(A) tail.

Oocytes were next injected with six individual brain mRNAs, which was followed by progesterone treatment, RNA extraction, chromatography on poly $(\mathrm{U})$ agarose, and detection by RT-PCR (the $3^{\prime}$ UTRs of these RNAs were too long to observe electrophoretic size shifts if they acquired poly(A) tails). Figure 2B shows that while the RNAs were detected in unfractionated (total) RNA from all oocytes, they were detected in the eluted fraction from poly(U) agarose only when the oocytes were treated with progesterone, thus indicating that they underwent hormone-induced polyadenylation.

To determine whether the RNAs selected as having undergone polyadenylation in a CPE-independent manner were true or false positives, several were cloned, synthesized in vitro, and injected independently into oocytes. While the positive control CPE-containing $\alpha$ CaMKII RNA indeed underwent polyadenylation following progesterone stimulation (Fig. 1), none of the CPE-lacking RNAs, CKB (creatine kinase B), cell-cell adhesion molecule 4 (C-CAM4), P2X2-3 ligand-gated channel (P2X2), and $\gamma$ tubulin $(\gamma \mathrm{Tub})$ did so (Fig. 2C). Thus, these RNAs were false positives in our assay.
We also examined whether an apparent CPE-containing RNA that was not selected in our assay was a false negative. PDE1 RNA was injected into oocytes that were subsequently stimulated by progesterone. Figure $2 \mathrm{C}$ shows that this RNA did not undergo polyadenylation. Thus, this RNA is a true negative, and this underscores the need for examining polyadenylation experimentally as opposed to predictions based solely on bioinformatics.

To assess whether RNAs undergo polyadenylation in an activity-dependent manner, hippocampal neurons cultured for 14-20 d were treated with glutamate, $\mathrm{KCl}$, or kainate before total RNA was extracted. While all selected RNAs were detected in the unfractionated (total) RNA, only $A B P$ (AMPA receptor binding protein), ELK2 (potassium channel), $\delta$ CaMKII, Map2, RCM3 (calmodulin), and $\alpha$ CaMKII mRNAs were found in the material thermally eluted from poly $(\mathrm{U})$ agarose, indicating that they became polyadenylated in response to synaptic activity (Fig. 3A). NF (neurofilament) RNA was retained on the column under all conditions, indicating that it neither gained nor lost a poly(A) tail in response to synaptic stimulation (Wu et al. 1998; Huang et al. 2002).

Finally, we addressed whether the proteins encoded by two of the mRNAs noted above underwent a commensurate increase in steady state levels in isolated synaptoneurosomes treated with glutamate (Fig. 3B). Compared to untreated controls, Map2 increased by about 35\% while ABP (the multiple bands probably represent different forms of ABP; Srivastava et al. 1998) increased by $72 \%$ (both increases are statistically significant, $p<0.05$, Student's $t$-test). Synaptophysin, a presynaptic marker for synaptoneurosomes, did not change in amount and served as an internal control. These results demonstrate that not only do several mRNAs undergo activity-dependent polyadenylation, but that this process is likely to promote increased rates of translation.

\section{DISCUSSION}

We have developed a method to identify brain mRNAs that undergo activity-dependent polyadenylation. The method is based on the observations that Xenopus oocytes and neurons recognize the same cis regulatory elements in RNA that control cytoplasmic polyadenylation, exploit the same basic polyadenylation machinery, and respond to extracellular signals that stimulate cytoplasmic polyadenylation (Wu et al. 1998; Huang et al. 2002). While these signals are different between the two cell types, progesterone in the case of Xenopus oocytes and NMDA (and agonists) in the case of neurons, they appear to converge at or upstream of Aurora A, a kinase that phosphorylates and activates $\mathrm{CPEB}$, an RNA binding protein that determines which mRNAs undergo polyadenylation. Xenopus oocytes are also biochemically facile, thus allowing them to serve as useful surrogates for examining $3^{\prime}$ end processing of heterologous mRNAs. 
TABLE 1. Results from microarray analysis

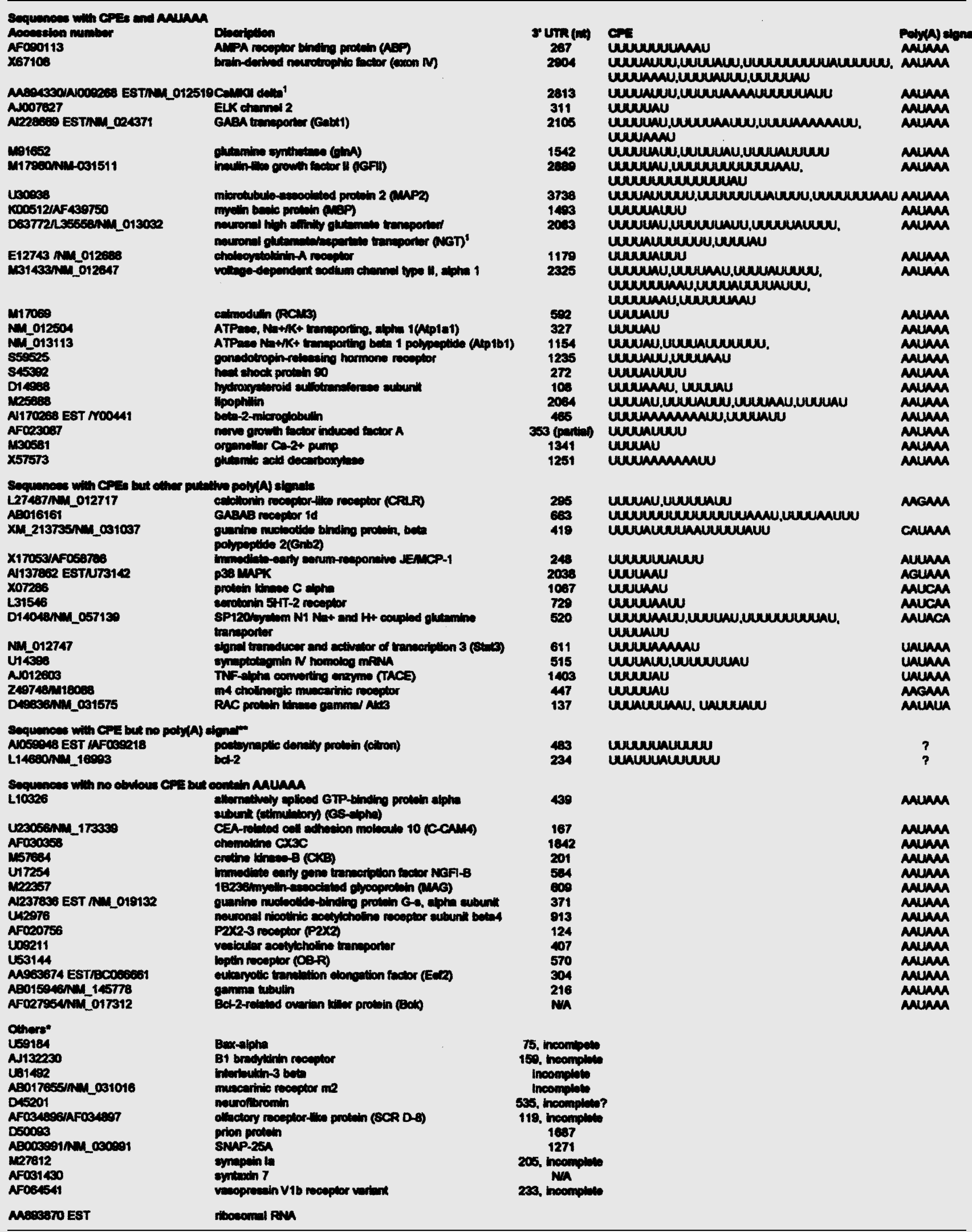

Note: ${ }^{1}$ These sequences have been detected twice.

*This group includes sequences with no obvious CPE and AAUAAA, or $3^{\prime}$ UTR is not complete or not available in database.

**The lack of poly(A) signal may also reflect the incompleteness of the $3^{\prime}$ UTR.

(NA) not available. 
TABLE 2. Summary of results from microarray analysis

- 25 pools containing 23,300 colonies

- 1200 sequences on rat neurobiology chip*

- 447 sequences from the initial probe are present on the chip

- 66 sequences were scored as having acquired a poly(A) tail in the oocyte injection assay

Number of sequences

(\% of total)

3' UTR

$\begin{array}{ll}39(59 \%) & \text { Contains CPE(s) } \\ 25 & \text { CPE and AAUAAA } \\ 12 & \text { CPE and other putative poly(A) signal } \\ 2 & \text { CPE but no polyadenylation signal** } \\ 14(21 \%) & \text { no CPE but contain AAUAAA } \\ 12(18 \%) & 3^{\prime} \text { UTR not available or incomplete } \\ 1(1 \%) & \text { ribosomal RNA }\end{array}$

*Affymetrix RN U34.

**Lack of AAUAAA may reflect incomplete 3' UTR.

We found that two methods used to measure poly(A) tail length, the PAT assay (Salles et al. 1994), which requires the generation of a nested series of oligo(dT)-poly(A) hybrids, and RNA circularization (Liu and Gorovsky 1993), which requires single-stranded RNA ligation, were inadequate for our screening purposes. Conversely, chromatography on poly $(\mathrm{U})$ agarose followed by thermal elution and detection by RT-PCR proved to be an efficient method for isolating RNA with poly (A) tails of greater than $\sim 55$ bases. However, this method does not discriminate among poly(A) tail lengths over a broad size range nor does it indicate the percentage of RNAs that have a poly $(\mathrm{A})$ tail of greater than $\sim 55$ bases (Simon et al. 1996). These can be important parameters to measure: For example, consider two populations of mRNA, one with an average poly(A) tail length of, say, 60 bases and another with an average poly(A) tail length of $\sim 100$ bases. While our method would not distinguish between these two populations, the RNAs with the long poly(A) tails would probably be translated at a greater rate since they would be bound by more molecules of poly $(\mathrm{A})$ binding protein, which through interactions with eIF4G, enhance initiation (Wakiyama et al. 2000). On the other hand, data from Xenopus oocytes show that the conversion of silent to active mRNAs requires that poly $(\mathrm{A})$ tails of $\sim 20-40$ bases be lengthened to $\sim 80$ 100 bases (Mendez and Richter 2001). These results are consistent with the poly(A) tail length changes and translational activation we observe in neurons in response to synaptic stimulation.
In our screen, we found that $\sim 15 \%$ (66 out of 447 ) of injected mRNAs underwent progesterone-induced polyadenylation. Of these, only $61 \%$ have obvious CPEs; $17 \%$ of the sequences do not have complete $3^{\prime}$ UTRs, so they may also have CPEs. Only $21 \%$ probably have complete $3^{\prime}$ UTRs (i.e., they contain AAUAAA) but no CPEs, at least based on the consensus structure (UUUU UAU). Most, if not all, the CPElacking RNAs probably represent false positives (Fig. 2C), and thus only $\sim 7 \%$ of RNAs probably truly undergo polyadenylation. This value could be even lower since the RNAs whose $3^{\prime}$ UTRs were not complete were not tested further.

We determined whether several mRNAs that underwent polyadenylation in injected oocytes also did so in neurons following synaptic stimulation. On the eight that were tested (based on their potential involvement in synaptic

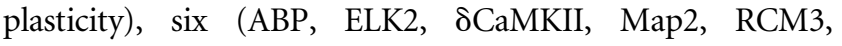
$\alpha$ CaMKII) became polyadenylated in an activity-dependent manner; two (Gabt1 and IGFII), however, did not (data not shown). Perhaps Gabt and IGFII mRNAs and CPEB reside in different subcellular compartments that make them refractory to polyadenylation. While we do not know whether the polyadenylation of the other six mRNAs occurred in the synapto-dendritic compartment, all the proteins encoded by them (ABP, $\delta$ CaMKII, $\alpha$ CaMKII, RCM3/calmodulin, Map2) are found in dendrites, suggesting the possibility that they are made locally. While ELK2 is not known to be dendritic, other voltage-gated $\mathrm{K}+$ channels related to ELK2 (Warmke and Ganetzky 1994) have been found in dendrites and at synapses (Johnston et al. 2003). Most importantly, we found that the proteins encoded by two of the mRNAs, Map2 and ABP, are synthesized in synaptoneurosomes treated with glutamate.

NMDA, but not AMPA or DHPG (metabotropic glutamate receptor agonist), stimulates $\mathrm{CPEB}$ phosphorylation

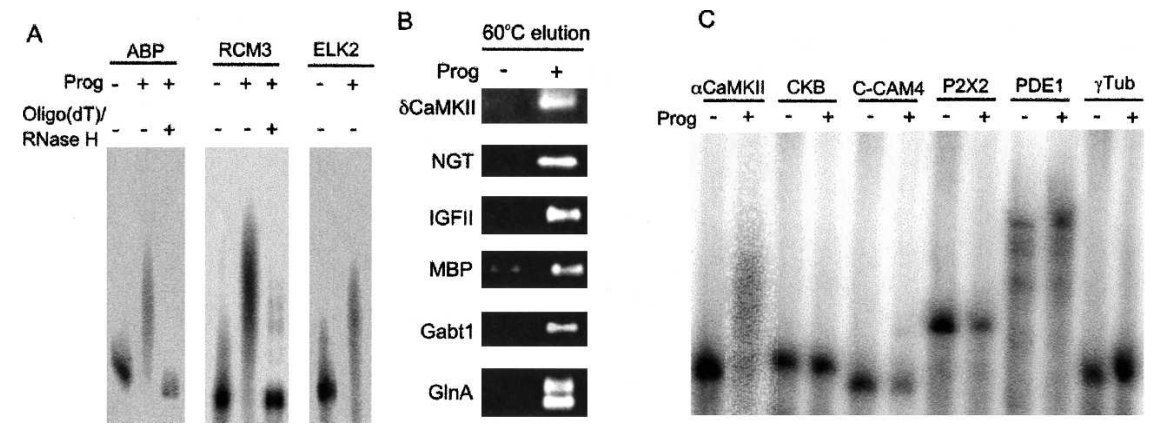

FIGURE 2. Brain RNAs undergo polyadenylation in injected Xenopus oocytes. (A) $3^{\prime}$ UTRs derived from ABP (AMPA binding protein), RCM3 (calmodulin), and ELK2 (ether a-go-go like, a potassium channel) mRNAs were synthesized in the presence of ${ }^{32} \mathrm{P}$-UTP and injected into oocytes, some of which were incubated with progesterone. Total RNA was extracted, some of which was treated with oligo(dT) and RNase $\mathrm{H}$ and then analyzed by denaturing PAGE. $(B)$ $3^{\prime}$ UTRs derived from $\delta$ CaMKII, NGT (neuronal glutamate transporter), IGFII (insulin like growth factor II), MBP (myelin basic protein), Gabt1 (GABA transporter), and GlnA (glutamine synthetase) were injected into oocytes and analyzed for polyadenylation as in Figure 1. (C) $3^{\prime}$ UTRs derived from $\alpha$ CaMKII, CKB (creatine kinase B), C-CAM4 (cell-cell adhesion molecule 4), P2X2 (receptor for ligated-gated channel), PDE1 (phosphodiesterase 1), and $\gamma$ tubulin $(\gamma \mathrm{Tub})$ were injected into oocytes an examined for polyadenylation as in Figure 1. 


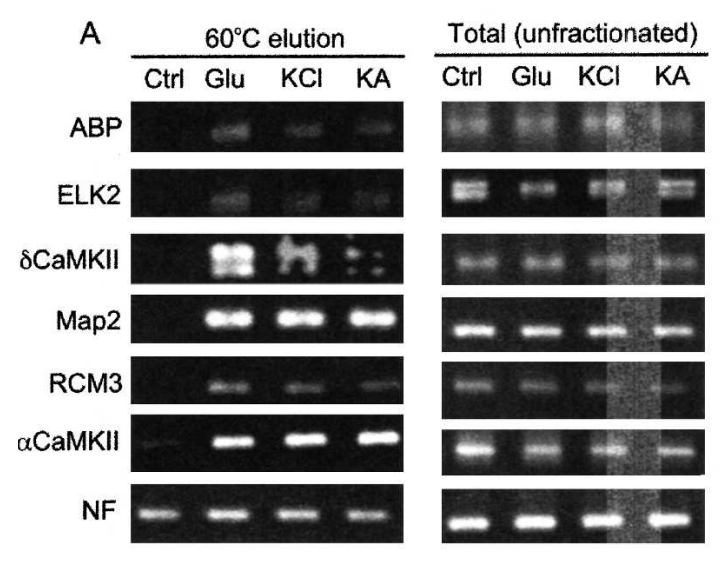

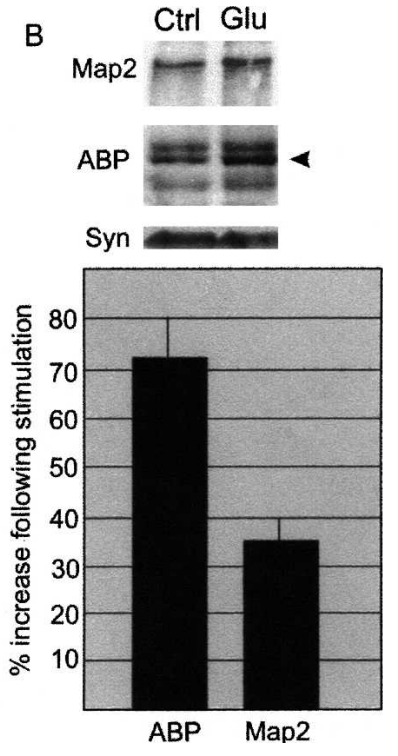

FIGURE 3. Activity-dependent polyadenylation in neurons. (A) Cultured hippocampal neurons were untreated or stimulated with glutamate, $\mathrm{KCl}$, or kainate. RNA was extracted and examined for polyadenylation as in Figure 1. (B) Protein from untreated synaptoneurosomes or those stimulated with glutamate for 10 min were Western blotted and probed for Map2, $\mathrm{ABP}$, and synaptophysin. The average values from three experiments, \pm SEM, are shown; the differences between the Map2 and ABP protein levels \pm glutamate were statistically significant $(p \leq 0.05$, Student's $t$-test).

and polyadenylation (Huang et al. 2002). This study shows that kainate also stimulates polyadenylation, and we would surmise CPEB phosphorylation as well. In this regard, it is worth noting that kainate, which binds to its own class of ionotropic glutamate receptors (Frerking and Nicoll 2000), stimulates $\alpha$ CaMKII and phosphorylated $\alpha$ CaMKII immunoreactivity in the CA3 region of the hippocampus; such events are blocked by cyclohexamide (Lee et al. 2003). It may be that the kainate-induced increase of $\alpha$ CaMKII occurred through CPEB and cytoplasmic polyadenylation. Unfortunately, we could not definitively show that CPEB is essential for polyadenylation since an insufficient number of cultured neurons could be obtained from CPEB knockout mice. Thus, the corresponding RNA levels were very low (data not shown). It is also worth noting that Shin et al. (2004) demonstrated that metabotropic glutamate receptor (mGluR) activation induced the polyadenylation of tPA mRNA, perhaps suggesting that CPEB can be activated through several signaling pathways.

This study indicates that many additional mRNAs undergo activity-dependent polyadenylation in neurons. If polyadenylation always leads to translation, which by no means is certain, then there may be a large number of newly synthesized proteins at synapses following stimulation. The screen described here will allow us to identify the mRNAs encoding such proteins, which may help elucidate the nature of protein-synthesisdependent synaptic plasticity. In addition, the screen we describe should be useful for identifying the panoply of RNAs that undergo polyadenylation in other systems, particularly where the amount of material that can be obtained is relatively low, such as maturing mouse oocytes.

\section{MATERIALS AND METHODS}

\section{Injection of oocytes with mRNA derived from a rat brain cDNA library}

Five hundred to 1000 bacterial clones derived from an oligo(dT)-primed and directionally cloned rat brain cDNA library (Invitrogen) were grown on 25 plates. Plasmid DNA was isolated from each plate and linearized with NotI, which is downstream of the A-T base pairs derived from reverse transcription of the brain RNA. To remove the poly(A) tails, the RNAs were treated with oligo(dT) and RNase $\mathrm{H}$ and phenol extracted. The RNAs $(\sim 0.1-0.2 \mu \mathrm{g} / \mu \mathrm{L})$ were injected into stage VI Xenopus oocytes (46.6 nL/ oocyte), which had been collected from ovaries treated with collagenase and dispase. Some oocytes were cultured in Barth's medium containing progesterone $(10 \mu \mathrm{M})$, which induces the oocytes to re-enter the meiotic divisions and progress into M-phase from a G2-like phase. One manifestation of oocyte maturation is the appearance of a white spot at the animal pole, indicating nuclear envelope (germinal vesicle) breakdown. The RNA was then extracted from both immature and mature oocytes.

\section{Selection of polyadenylated RNA and microarray analysis}

The RNA extracted from oocytes was denatured on poly(U) agarose (Sigma) in buffer containing 25\% formamide, $0.1 \mathrm{M} \mathrm{NaCl}, 50$ $\mathrm{mM}$ Tris- $\mathrm{HCl}$ (pH 7.5), and $10 \mathrm{mM}$ EDTA as described by Simon et al. (1996). The matrix was washed with several bed volumes of chromatography buffer at room temperature and then at $50^{\circ} \mathrm{C}$; the RNA was eluted at $60^{\circ} \mathrm{C}$. Simon et al. (1996) estimated that poly(A) length of $\geq 55 \mathrm{nt}$ will remain on the column at $50^{\circ} \mathrm{C}$ but be eluted at $60^{\circ} \mathrm{C}$. The poly(A) RNA from each pool was combined and used as template to synthesize biotin-labeled cRNA probe that was hybridized to the Affymetrix rat neurobiology microarray chip (RN U34), which was carried out in the Gene Microarray Facility at the University of Massachusetts Medical School. Images were acquired using a GeneArray scanner (Agilent Technologies) and analyzed with Microarray Suite 5.0 software (MAS 5) (Affymetrix).

\section{Confirmation of polyadenylation}

Two methods were used to confirm RNA polyadenylation in oocytes. For the first, RNAs fewer than $\sim 200$ nt in length were synthesized in vitro by T7 polymerase in the presence of ${ }^{32} \mathrm{P}^{\prime}$-UTP (Mendez et al. 2000) and then injected into oocytes, some of which were incubated with progesterone. The RNAs were then extracted 
and a portion of the sample was annealed to oligo(dT) and digested with RNase $\mathrm{H}$ (Invitrogen) to remove the poly(A) tail before analysis on denaturing 5\% polyacrylamide gels. Longer RNAs were synthesized in vitro with a mMessage mMachine high yield capped RNA transcription kit (Ambion) and injected into oocytes, some of which were treated with progesterone. Total RNA was isolated and subjected to chromatography on poly(U) agarose described above; RT-PCR with sequence-specific primers was used to detect the RNAs.

\section{Cell culture and synaptosome preparation}

Rat hippocampal neurons were prepared and cultured as described (Banker and Goslin 1988). Cells maintained in neuron basal medium (supplemented with B27) for 14-20 d were stimulated with $0.3 \mathrm{mM}$ glutamate $/ 10 \mu \mathrm{M}$ glycine or $300 \mu \mathrm{M}$ kainic acid for 15 min or $50 \mathrm{mM} \mathrm{KCl}$ for $5 \mathrm{~min}$, then incubated for 10 more minutes before RNA extraction by the TRIZol reagent (Invitrogen). Polyadenylation was then assayed by thermal elution from poly $(\mathrm{U})$ agarose and RT-PCR as noted above.

Synaptosomes were prepared from rat whole brain extract using a sucrose-Percoll discontinuous gradient as described previously (Bagni et al. 2000; Huang et al. 2002). They were suspended in buffer containing a protease inhibitor cocktail (Sigma) and stimulated with $0.3 \mathrm{mM}$ glutamate $/ 10 \mu \mathrm{M}$ glycine for $10 \mathrm{~min}$ before being processed for Western blots probed with antibodies for ABP (Chemicon), Map2 (Sigma), and synaptophysin (Chemicon) at 1:500 dilution. Equal loading of the gels was confirmed by Ponceau-S staining.

\section{ACKNOWLEDGMENTS}

L.D. was supported by an institutional postdoctoral training grant (HD07312). This work was supported by a grant from the $\mathrm{NIH}$ (NS39321). Additional funding from the Diabetes Endocrinology Research Center (DK32520) is gratefully acknowledged.

Received April 21, 2005; accepted June 2, 2005.

\section{REFERENCES}

Aakalu, G., Smith, W.B., Nguyen, N., Jiang, C., and Schuman, E.M. 2001. Dynamic visualization of local protein synthesis in hippocampal neurons. Neuron 30: 489-502.

Alarcon, J.M., Hodgman, R., Theis, M., Huang, Y-S., Kandel, E.R., and Richter, J.D. 2004. Selective modulation of some forms of Schaffer collateral-CA1 synaptic plasticity in mice with a disruption of the CPEB-1 gene. Learn. Mem. 11: 318-327.

Atkins, C.M., Nozaki, N., Shigeri, Y., and Soderling, T.R. 2004. Cytoplasmic polyadenylation element binding protein-dependent protein synthesis is regulated by calcium/calmodulin-dependent protein kinase II. J. Neurosci. 24: 5193-5201.

Bagni, C., Mannucci, L., Dotti, C.G., and Amaldi, F. 2000. Chemical stimulation of synaptosomes modulates $\alpha-\mathrm{Ca} 2+/$ calmodulindependent protein kinase II mRNA association to polysomes. $J$. Neurosci. 20: RC76.

Banker, G. and Goslin, K. 1988. Developments in neuronal cell culture. Nature 336: 185-186.

Cao, Q. and Richter, J.D. 2002. Dissolution of the maskin-eIF4E complex by cytoplasmic polyadenylation and poly(A) binding protein controls cyclin B1 mRNA translation and oocyte maturation. EMBO J. 21: 3852-3862.
Frerking, M. and Nicoll, R.A. 2000. Synaptic kainate receptors. Curr. Opin. Neurobiol. 10: 342-351.

Frey, U. and Morris, R.G. 1997. Synaptic tagging and long-term potentiation. Nature 385: 533-536.

Frey, U., Muller, M., and Kuhl, D. 1996. A different form of longlasting potentiation revealed in tissue plasminogen activator mutant mice. J. Neurosci. 16: 2057-2063.

Hering, H. and Sheng, M. 2001. Dendritic spines: Structure, dynamics and regulation. Nat. Rev. Neurosci. 2: 880-888.

Huang, Y.-S., Jung, M.-Y., Sarkissian, M., and Richter, J.D. 2002. Nmethyl- $D$-aspartate receptor signaling results in aurora kinasecatalyzed CPEB phosphorylation and $\alpha$ CaMKII mRNA polyadenylation at synapses. EMBO J. 21: 2139-2148.

Huber, K.M., Kayser, M.S., and Bear, M.F. 2000. Role for rapid dendritic protein synthesis in hippocampal mGluR-dependent long-term depression. Science 288: 1254-1257.

Johnston, D., Christie, B.R., Frick, A., Gray, R., Hoffman, D.A., Schexnayder, L.K., Watanabe, S., and Yuan, L.L. 2003. Active dendrites, potassium channels and synaptic plasticity. Phil. Trans. R. Soc. Lond. B Biol. Sci. 358: 667-674.

Kang, H. and Schuman, E.M. 1996. A requirement for local protein synthesis in neurotrophin-induced hippocampal synaptic plasticity. Science 273: 1402-1406.

Lee, H.K., Choi, S.S., Han, E.J., Han, K.J., and Suh, H.W. 2003. Role of glutamate receptors and an on-going protein synthesis in the regulation of phosphorylation of $\mathrm{Ca} 2+/$ calmodulin-dependent protein kinase II in the CA3 hippocampal region in mice administered with kainic acid intracerebroventricularly. Neurosci. Lett. 348: 93-96.

Liu, X. and Gorovsky, M.A. 1993. Mapping the $5^{\prime}$ and $3^{\prime}$ ends of Tetrahymena thermophila mRNAs using RNA ligase mediated amplification of cDNA ends (RLM-RACE). Nucleic Acids Res. 21: 4954-4960.

Martin, K.C., Casadio, A., Zhu, H., Yaping, E., Rose, J.C., Chen, M., Bailey, C.H., and Kandel, E.R. 1997. Synapse-specific, long-term facilitation of Aplysia sensory to motor synapses: A function for local protein synthesis in memory storage. Cell 91: 927-938.

Mendez, R. and Richter, J.D. 2001. CPEB-mediated translation: A means to the end. Nat. Rev. Mol. Cell. Biol. 2: 521-530.

Mendez, R., Hake, L.E., Andresson, T., Littlefield, L.E., Ruderman, J.V., and Richter, J.D. 2000. Phosphorylation of CPE binding factor by Eg2 regulates c-mos mRNA translation. Nature 404: 302-307.

Miller, S. and Mayford, M. 1999. Cellular and molecular mechanisms of memory: The LTP connection. Curr. Opin. Genet. Dev. 9: 333-337.

Palatnik, C.M., Storti, R.V., and Jacobson, A. 1981. Partial purification of a developmentally regulated messenger from Dictyostelium discoideum by thermal elution from poly(U)-Sepharose. J. Mol. Biol. 150: $389-398$.

Richter, J.D. 2001. Think globally, translate locally: What mitotic spindles and mammalian neurons have in common. Proc. Natl. Acad. Sci. 98: 7069-7071.

Salles, F.J., Lieberfarb, M.E., Wreden, C., Gergen, J.P., and Strickland, S. 1994. Coordinate initiation of Drosophila development by regulated polyadenylation of maternal messenger RNAs. Science 266: 1996-1999.

Scheetz, A.J., Nairn, A.C., and Constantine-Paton, M. 2000. NMDA receptor-mediated control of protein synthesis at developing synapses. Nat. Neurosci. 3: 211-216.

Shin, C.Y., Kundel, M., and Wells, D.G. 2004. Rapid, activity-induced increase in tissue plasminogen activator is mediated by metabotropic glutamate receptor-dependent mRNA translation. J. Neurosci. 24: 9425-9433.

Si, K., Giustetto, M., Etkin, A., Hsu, R., Janisiewicz, A., Conchetta, M., Kim, M., Zhu, H., and Kandel, E.R. 2003. A neuronal isoform of CPEB regulates local protein synthesis and stabilizes synapse-specific longterm facilitation in Aplysia. Cell 115: 893-904.

Silva, A.J., Stevens, C.F., Tonegawa, S., and Wang, Y. 1992. Deficient hippocampal long-term potentiation in $\alpha$-calcium-calmodulin kinase II mutant mice. Science 257: 201-206. 
Simon, R., Wu, L., and Richter, J.D. 1996. Cytoplasmic polyadenylation of activin receptor mRNA and the control of pattern formation during Xenopus development. Dev. Biol. 179: 239250.

Srivastava, S., Osten, P., Vilim, F.S., Khatri, L., Inman, G., States, B., Daly, C., DeSouza, S., Abagyan, R., Valtschanoff, J.G., et al. 1998. Novel anchorage of GluR2/3 to the postsynaptic density by the AMPA receptor-binding protein ABP. Neuron 21: 581-591.

Stebbins-Boaz, B., Cao, Q., deMoor, C., Mendez, R., and Richter, J.D. 1999. Maskin is a CPEB-associated factor that transiently interacts with eIF-4E. Mol. Cell 4: 1017-1027.
Wakiyama, M., Imataka, H., and Sonenberg, N. 2000. Interaction of eIF4G with poly(A)-binding protein stimulates translation and is critical for Xenopus oocyte maturation. Curr. Biol. 10: 1147-1150.

Warmke, J.W. and Ganetzky, B. 1994. A family of potassium channel genes related to eag in Drosophila and mammals. Proc. Natl. Acad. Sci. 91: 3438-3442.

Wu, L., Wells, D., Tay, J., Mendis, D., Abbot, M., Barnitt, A., Quinlan, E., Heynen, A., Fallon, J., and Richter, J.D. 1998. CPEB-mediated cytoplasmic polyadenylation and the regulation of experiencedependent translation of $\alpha$-CaMKII mRNA at synapses. Neuron 21: 1129-1139. 